\title{
BMC
}

Structural Biology

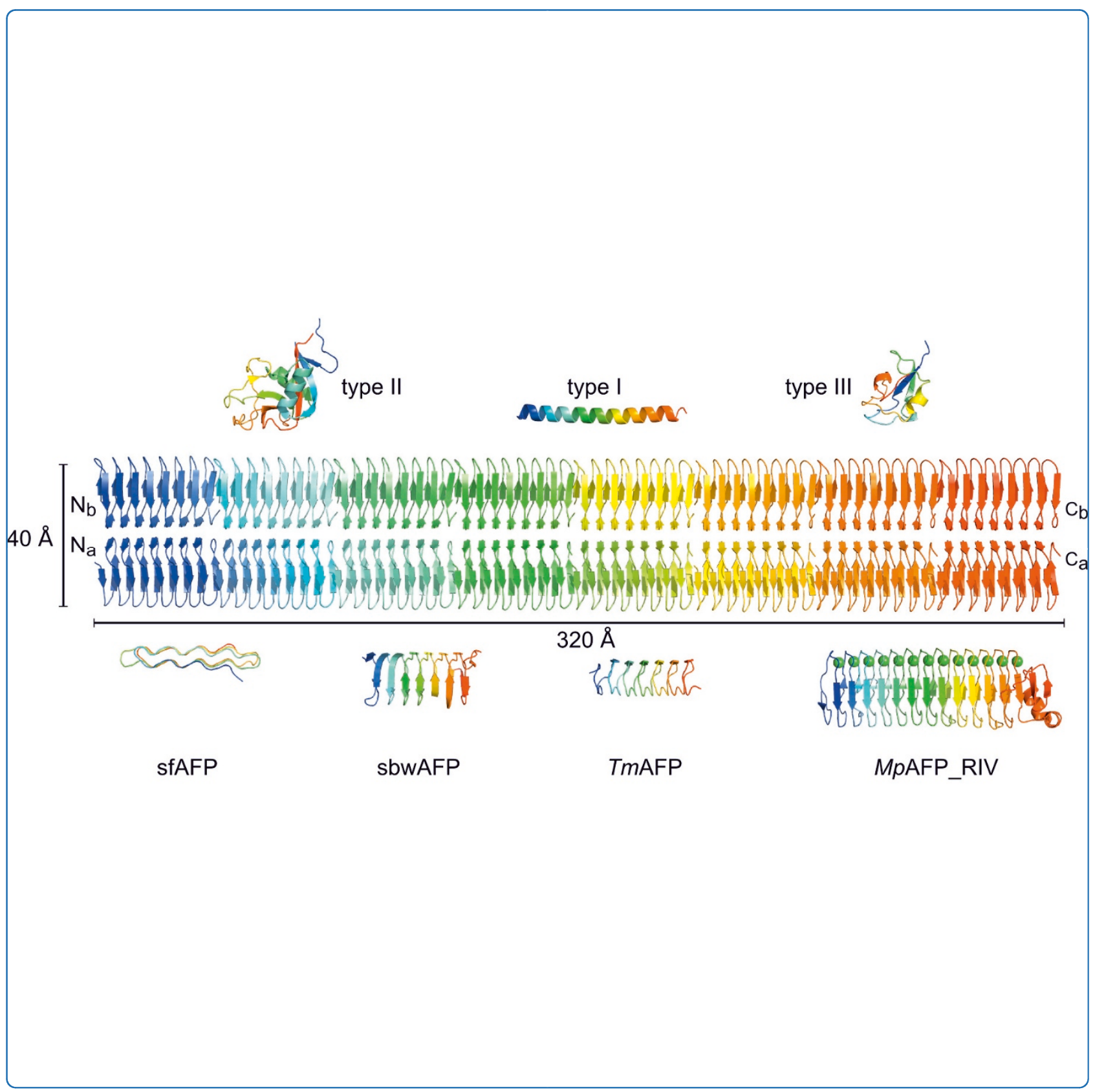

\section{Novel dimeric $\beta$-helical model of an ice nucleation protein with bridged active sites}

Garnham et al. 


\title{
Novel dimeric $\beta$-helical model of an ice nucleation protein with bridged active sites
}

\author{
Christopher P Garnham ${ }^{1}$, Robert L Campbell', Virginia K Walker ${ }^{2,3}$ and Peter L Davies ${ }^{1,2^{*}}$
}

\begin{abstract}
Background: Ice nucleation proteins (INPs) allow water to freeze at high subzero temperatures. Due to their large size $(>120 \mathrm{kDa})$, membrane association, and tendency to aggregate, an experimentally-determined tertiary structure of an INP has yet to be reported. How they function at the molecular level therefore remains unknown.

Results: Here we have predicted a novel $\beta$-helical fold for the INP produced by the bacterium Pseudomonas borealis. The protein uses internal serine and glutamine ladders for stabilization and is predicted to dimerize via the burying of a solvent-exposed tyrosine ladder to make an intimate hydrophobic contact along the dimerization interface. The manner in which PbINP dimerizes also allows for its multimerization, which could explain the aggregation-dependence of INP activity. Both sides of the PbINP structure have tandem arrays of amino acids that can organize waters into the ice-like clathrate structures seen on antifreeze proteins.

Conclusions: Dimerization dramatically increases the 'ice-active' surface area of the protein by doubling its width, increasing its length, and presenting identical ice-forming surfaces on both sides of the protein. We suggest that this allows sufficient anchored clathrate waters to align on the INP surface to nucleate freezing. As PbINP is highly similar to all known bacterial INPs, we predict its fold and mechanism of action will apply to these other INPs.
\end{abstract}

\section{Background}

Two extraordinary families of proteins have evolved to influence ice growth in opposite ways: antifreeze proteins (AFPs) that irreversibly adsorb to the surface of ice crystals to prevent their further growth [1]; and icenucleation proteins (INPs) that cause ice to form in solution at high sub-zero temperatures $[2,3]$. Whereas AFPs are small (Mr 3,000 - 35,000), and generally monomeric proteins, INPs are large $(\mathrm{Mr}>100,000)$ and function as multimers [4].

The tertiary structures of many AFPs are known, but none has been experimentally determined for an INP. Most INPs contain three distinct domains, with the majority of their mass residing within a highly repetitive central domain [5]. This domain consists of a variable number (ca. 50-80) of tandem 16-amino-acid (aa) repeats, with each repeat following the general consensus sequence of GYGSTxTAxxxSxLxA [6]. NMR and CD studies of synthetic bacterial INP peptides have not

\footnotetext{
* Correspondence: peter.davies@queensu.ca

'Department of Biochemistry, Queen's University, Kingston, ON, K7L-3N6, Canada Full list of author information is available at the end of the article
}

revealed a basic folding unit [6-9]. Molecular models of INP from the bacterium Pseudomonas syringae have included a planar array of anti-parallel $\beta$-strands [10] and a left-handed $\beta$-helix [6]. The latter was modelled on UDP-acetylglucosamine acyltransferease as an AFPlike $\beta$-helix with the $\beta$-stranded TxT motifs located within each 16-aa repeat aligning down one side of the protein and functioning as the site of ice nucleation. Interestingly, a 96-aa recombinantly-expressed fragment of PsINP was shown to produce moderate levels of AFP activity [11], hinting that INPs and AFPs share a similar mechanism of action.

The Gram-negative bacterium $P$. borealis produces a 1244-aa INP (PbINP) similar to all other known bacterial INPs [12]. Here we have predicted the structure of a 128-aa segment of the protein using a combination of homology-based modelling and molecular dynamics (MD) simulations. The right-handed $\beta$-helical model is stabilized by internal serine and glutamine ladders, and is predicted to dimerize via a highly conserved solventexposed tyrosine ladder. ach chain of the dimer contains two putative ice-nucleation sites, located opposite one another, and comprised of repetitive TQTA and SLTA
C Biomed Central 
$\beta$-strands. Each surface is flat and relatively hydrophobic, but also replete with hydrogen bond donors and acceptors; hallmarks of a typical AFP ice-binding site (IBS). MD simulations show each site is capable of ordering water molecules into an ice-like lattice, indicating that INPs use the same anchored clathrate water $(\mathrm{ACW})$ mechanism of action that was recently elucidated for all AFPs [13]. Indeed, ACWs align across the entire width of both sides of the INP dimer, dramatically increasing the 'active' surface area of the protein and further strengthening the idea that size, and not fold or mechanism of action, is the primary discriminating factor between the two families of proteins with diametrically opposite functions regarding ice growth.

\section{Results and Discussion}

\section{PbINP contains two ice-nucleating motifs per 16-aa} repeat

The highly repetitive central domain of $P b I N P$ consists of 64 tandem 16-aa repeats (Figure 1A). Each repeat contains four distinct tetra-peptide motifs; two putative $\beta$-strands with consensus sequences of TQTA and SLTA, and two glycine- and serine-rich turns with consensus sequences of xxxS and GYGS (Figure 1B). Each 16-aa repeat forms part of a higher order 48-aa repeat, consisting of three tandem 16-aa sequences (Figure 1C).

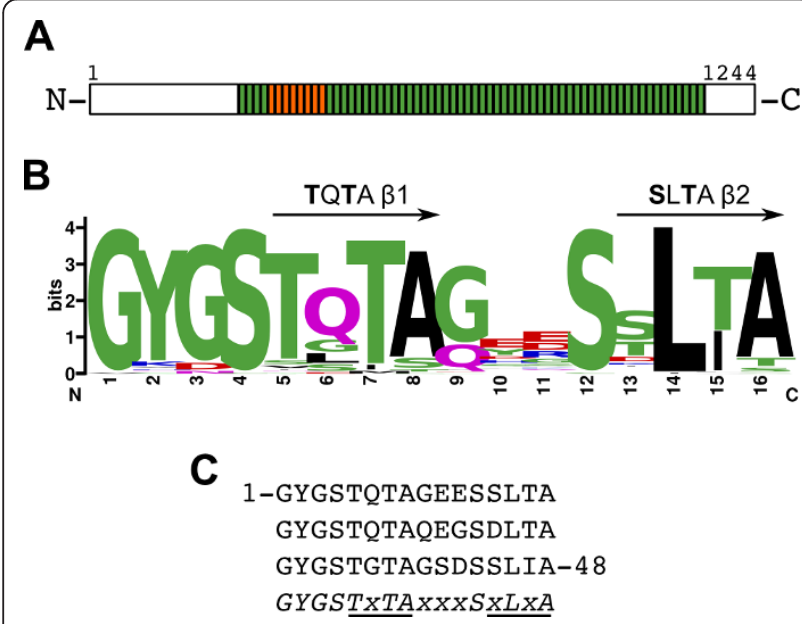

Figure 1 Schematic representation of PbINP and its repetitive amino acid sequence. A) Each green or orange box represents one 16-aa repeat within the repetitive central domain. The nonrepetitive $\mathrm{N}$ - and $\mathrm{C}$-terminal domains are indicated by white rectangles. Boxes coloured orange are the eight repeats modelled in this study. B) WEBLOGO plot [41] of the 64 16-aa repeats of PbINP. Hydrophobic residues are coloured black, acidic residues red, basic residues blue. Other residues are coloured green or purple. C) The amino acid sequence of PbINP residues 217-265 is shown. The consensus amino acid sequence of the $3 \times 16$-aa block is indicated below in italics. Putative $\beta$-strands are underlined while $x$ represents any amino acid.
The tetra-peptide TQTA and LTA $\beta$-strands (predicted solvent-exposed residues in bold) are highly similar to the TxT motifs that define the ice-binding sites (IBS) of $\beta$-helical insect and grass AFPs [14-17]. Previous modelling of the homologous $P$. syringae INP (PsINP) based on UDP-acetylglucosamine acyltransferase (PDB 1LXA) predicted each 16-aa repeat of the protein formed one loop of a left-handed $\beta$-helix [6]. This aligned the TQTA motifs down one side of he protein, forming a flat $\beta$-sheet that functioned as the site of ice nucleation. Since the 16-aa repeats of PbINP (and all sequenced bacterial INPs) contain two putative TxT-like $\beta$-strands separated by glycine- and serine-rich tetra-peptide turns, each strand of PbINP was predicted to align on opposite sides of a $\beta$-helical structure, forming two parallel $\beta$ sheets. For this reason, the $\beta$-roll from alkaline protease (PDB 1KAP) was chosen here as a starting template from which to model $\mathrm{PbINP}$. Located between its $\mathrm{Ca}^{2}$ ${ }^{+}$-binding turns are two parallel $\beta$-sheets packed against one another (Figure 2A). The model of PbINP was built by excising this central portion of the $\beta$-roll from alkaline protease and changing its residues to the appropriate TQTA and SLTA motifs (Figure 2B). The xxxS and GYGS loops were manually built to connect the strands (see Additional file 1, Figure S1A), and the newly built 16-aa loop was duplicated, substituted with the appropriate $P b I N P$ sequence, and aligned to the ensuing $\beta$ strands of the structure. This was performed until an eight-loop parallel $\beta$-helix was built that corresponds to residues 217-345 of bINP (see Additional file 1, Figure S1B).

\section{PbINP folds as a parallel $\beta$-helix}

PbINP was modelled as a right-handed $\beta$-helix due to the right-handedness of the modelling template. $M p$ AFP_RIV was previously modelled as a right-handed $\beta$ helix using the same $\beta$-roll template from alkaline protease and proved to be right-handed based on its crystal structure $[13,18]$. However, PbINP could just as likely be left-handed, and the handedness of an ice-binding protein does not affect its ability to bind ice. Both Land D-enantiomers of type I fish AFP and snowflea AFP display identical levels of antifreeze activity $[19,20]$, while the non-homologous $\beta$-helical insect AFPs produced by Tenebrio molitor [15] and Choristoneura fumiferana [14] are right- and left-handed respectively, yet display near identical ice-binding sites and activity levels. Therefore, while we modelled PbINP as a right-handed $\beta$-helix, we think that either a left- or right-handed version of the protein would be equally capable of efficiently nucleating ice.

To test the stability of the initial PbINP model, a solvated 5-ns MD simulation was performed. The model stabilized at the 2 -ns mark of the simulation and 


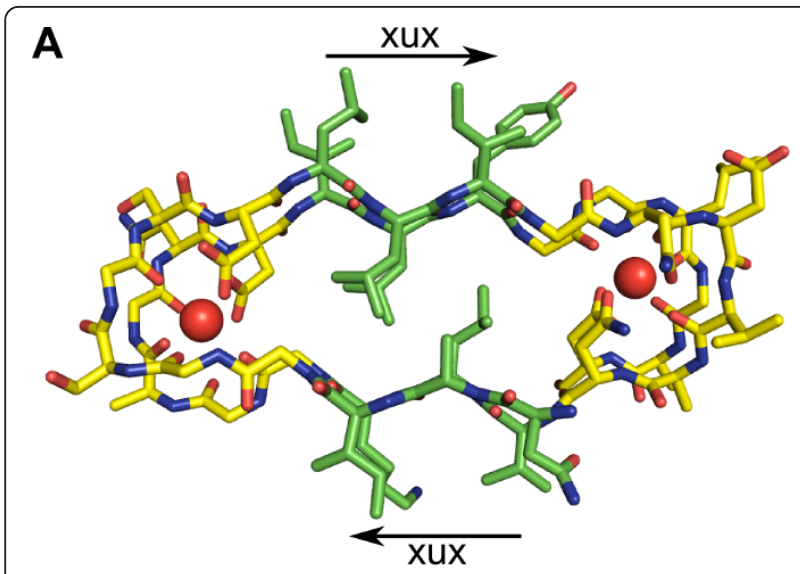

B

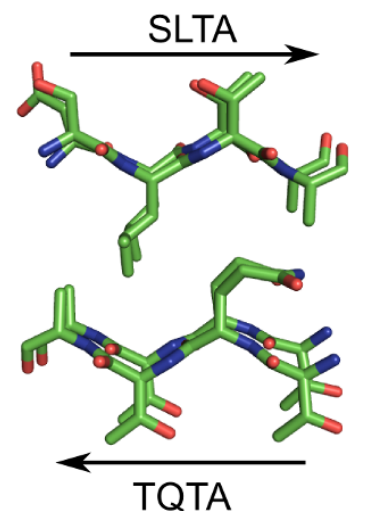

Figure 2 Modelling of PbINP. A) The $\beta$-roll (residues 330-348) from alkaline protease (PDB 1KAP) was used as the starting template. Carbon atoms from the central $\beta$-strands of the structure are coloured green, while carbons from the $\mathrm{Ca}^{2+}$-binding turns are coloured yellow. Oxygen atoms are coloured red, nitrogen atoms blue, and $\mathrm{Ca}^{2+}$ ions are shown as red spheres. Arrows indicate $\beta$ strands, with $\mathrm{x}$ representing any residue and $\mathrm{u}$ representing hydrophobic residues. B) The excised $\beta$-strands altered to conform to the sequence of PbINP. The consensus sequence of each strand is indicated.

remained remarkably constant throughout the remainder of the trajectory (see Additional file 1, Figure S2A). An energy-minimized average structure of the model was calculated from the simulation's final $3 \mathrm{~ns}$. The $\beta$ helix is roughly $40 \AA$ in length $\times 20 \AA$ wide $\times 5-10 \AA$ thick (Figure 3A,B). A Ramachandran plot of the structure shows that all residues fall within allowable regions (see Additional file 1, Figure S2B). The protein contains a modest hydrophobic core, consisting of the alanine methyl groups at the end of each $\beta$-strand (TQTA and SLTA), the leucine side chain from the SLTA $\beta$-strands, and the side-chain methylene groups of the inward-projecting glutamine and serine residues from the TQTA and $\operatorname{xxx}$ S motifs respectively (Figure 3B). The leucine residues interdigitate with the side-chain methylene groups of the glutamine residues, further increasing

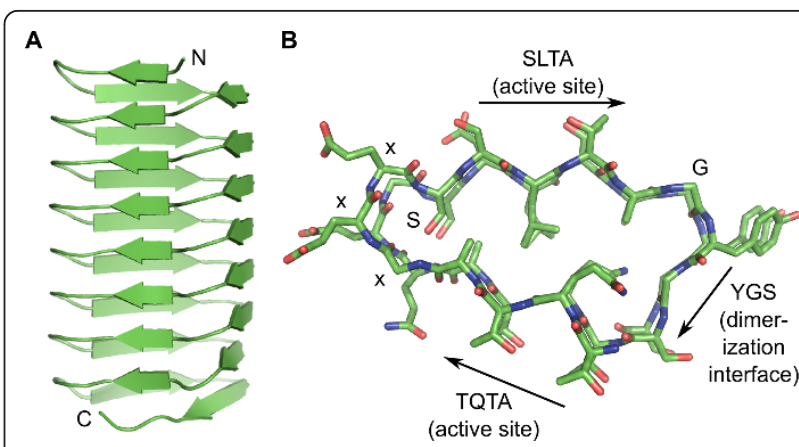

Figure $3 \boldsymbol{\beta}$-helical model of PbINP. A) Cartoon representation of the energy-minimized average structure of PbINP (residues 217-345) following a 5 -ns MD simulation. $\beta$-strands are indicated by green arrows. The $\mathrm{N}$ and $\mathrm{C}$ termini are indicated. B) Cross-sectional view of PbINP. The $\beta$-standed SLTA and TQTA active sites are indicated, as is the $\beta$-stranded YGS multimerization interface. Oxygen atoms are coloured red, nitrogen atoms blue, and carbon atoms green.

stabilization. The polar groups of the internal glutamine and serine residues point toward the turns of the protein and away from the hydrophobic core, and mainchain inter-loop hydrogen bonds shield the hydrophobic core from the solvent.

The inward-pointing serine and glutamine residues each form a ladder in opposite corners of the structure. The serines of the ladder reside in the final position of the $\operatorname{xxxS}$ motif that connects the TQTA $\beta$-strand to the SLTA $\beta$-strand (Figure 3B). Each Ser hydroxyl group hydrogen bonds to main-chain carbonyl oxygens and amide nitrogens of the same and ensuing xxxS motifs of the structure, while the main-chain amide nitrogen of each serine hydrogen bonds with the main-chain carbonyl oxygen of the second $\mathrm{x}$ residue of the $\mathrm{xxxS}$ motif of the ensuing loop (Figure 4A). The three $x$ residues of the $\mathrm{xxxS}$ motif adopt an $\mathrm{xbl} \beta$-arcade as defined by Hennetin et al. [21]. $\beta$-arcades of this configuration are typically followed by residues with short polar side chains that point inwards, allowing them to form stablizing H-bonds with the protein's main chain. This is clearly occurring in PbINP with the highly conserved inward-pointing row of Ser residues. Also, the hyperactive $\beta$-helical AFP from the beetle Tenebrio molitor (TmAFP) uses an internal serine ladder to stabilize turns within its structure [15].

The glutamine ladder is formed by the inward-pointing Gln residue of each TQTA motif (Figure 3B). Glutamines typically repeat two out of every three loops at this position, with the third loop usually having a glycine substitution. Glutamine side chains form hydrogen bonds with main-chain carbonyl oxygens and amide nitrogens of the turn immediately preceding the TQTA motif, as shown in stereo in Figure 4B. The void produced by the regularlyspaced glycine substitutions is filled by an internal water 

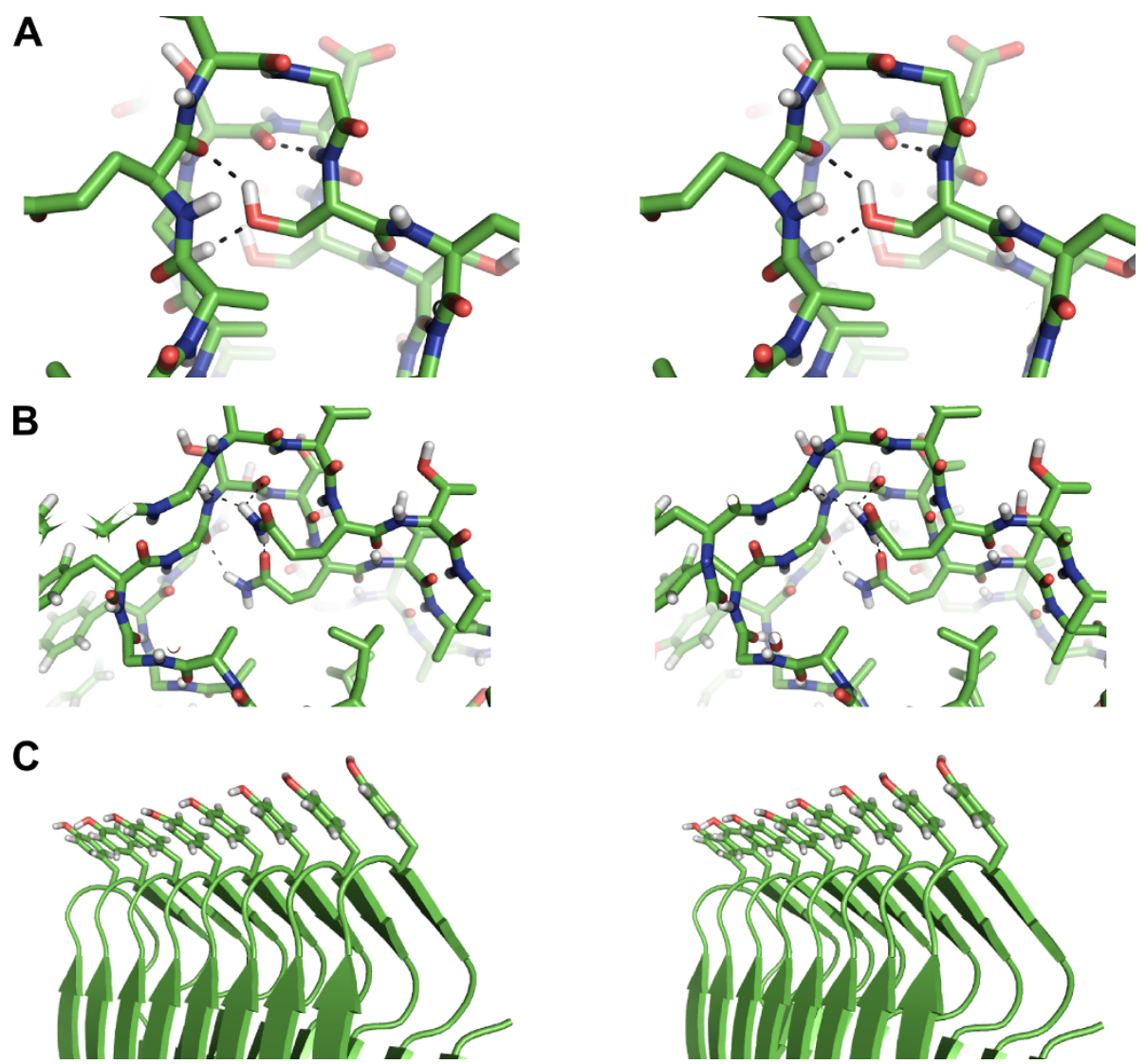

Figure 4 The serine, glutamine, and tyrosine ladders of PbINP. A) Stereo view of the internal serine ladder. The colouring scheme is the same as in Figure 3. Hydrogen bonds are represented by black dashed lines. Hydrogen atoms are coloured white. Each serine -OH group forms two hydrogen bonds; one to the main-chain carbonyl oxygen from the first x residue of the xxxS motif of the same loop, and one to the mainchain amide nitrogen of the first $x$ residue of the $\mathrm{x} x \mathrm{XS}$ motif of the ensuing loop. The main-chain amide of each serine residue also hydrogen bonds to the main-chain carbonyl oxygen of the second $x$ residue of the xxxS motif of the ensuing loop, further stabilizing the turn. B) Stereo view of the internal glutamine ladder. The side-chain amide of the glutamine forms hydrogen bonds with a side-chain carbonyl oxygen on the lower part of the loop, as well as with the main-chain carbonyl oxygen of the second glycine of the GYGS motif of the same loop and the serine of the GYGS motif of the ensuing loop. C) Stereo view of the external tyrosine ladder. The main chain of the protein is shown in cartoon format.

molecule that bridges adjacent glutamines and also forms hydrogen bonds to the main chain of the protein (see Additional file 1, Figure S3). The hyperactive $\beta$-helical TmAFP uses internal water molecules to stabilize its structure [15]. Here it should be noted that these proteins have evolved to function at sub-zero temperatures where hydrogen bonding is relatively strong. While no structure in the Protein Data Bank contains a glutamine ladder, several $\beta$-helical proteins use very similar asparagine ladders to stabilize their folds [22], and TibA, a 104-kDa bacterial glycoprotein with both adhesin and invasin properties, is also predicted to contain an internal glutamine ladder [23].

\section{PbINP dimerizes via a solvent-exposed tyrosine ladder} The tightly packed core of the protein left no room for the tyrosine residue of the GYGS motifs, and as such, they were forced to adopt a solvent-exposed orientation during initial model building. During the course of the MD simulation, the tyrosine residues stacked on top of one another (Figure 3B, Figure 4C), and each tri-peptide YGS motif adopted a $\beta$-stranded conformation. Stacking of solvent-exposed aromatic residues has been observed within various proteins, and they typically reside in areas involved in receptor binding and/or dimerization. For example, InIJ, a $\beta$-helical leucine-rich repeat produced by the bacterium Listeria monocytogenes, contains multiple solvent-exposed and stacked aromatic residues within its predicted receptor recognition domain [24]. Most interestingly, engineered tyrosine ladders have been shown to promote the flatness [25] and dimerization [26] of OspA, a single-layer- $\beta$-sheet containing protein from the bacterium Borrelia burgdorferi. These results hinted at the possibility that PbINP's tyrosine ladder might play a role in dimerization. 
To test this idea, a model dimer of PbINP was built by aligning two identical chains of PbINP in a parallel manner such that their tyrosine ladders were oriented towards one another and their $\mathrm{N}$ termini were at the same end of the structure. This placed the SLTA surface of one chain on the same side of the structure as the TQTA surface from the opposite chain. A 10-ns MD simulation was then performed where water molecules were allowed to equilibrate at the dimerization interface prior to the start of the trajectory (Figure 5A). Immediately following the start of the simulation, the two chains moved towards one another, driven by the desolvation of the aromatic rings of the tyrosine residues (Figure 5B). The dimeric structure stabilized at the 5-ns mark of the trajectory and remained completely stable thereafter (Figure 5C). The energy- minimized average dimeric structure is shown in Figure 6. All waters were excluded from the dimerization interface as the aromatic rings of each tyrosine residue packed tightly against the $\beta$-stranded glycine residues of the opposite chain's GYGS motif (Figure 5B). Interestingly, the hydroxyl group of each tyrosine formed part of an intercalated hydrogen-bond network with the side-chain hydroxyl groups of the outward-projecting serine residues of the opposite chain's GYGS motifs (Figure 6). This is exemplified by the number of side-chain hydrogen bonds that formed during the simulation, increasing from 7 at the start to an average of 20 upon stabilization of the dimerization interface (Figure 5D).

To test the stability of the dimer, 10-ns MD simulations were performed on the energy-minimized average
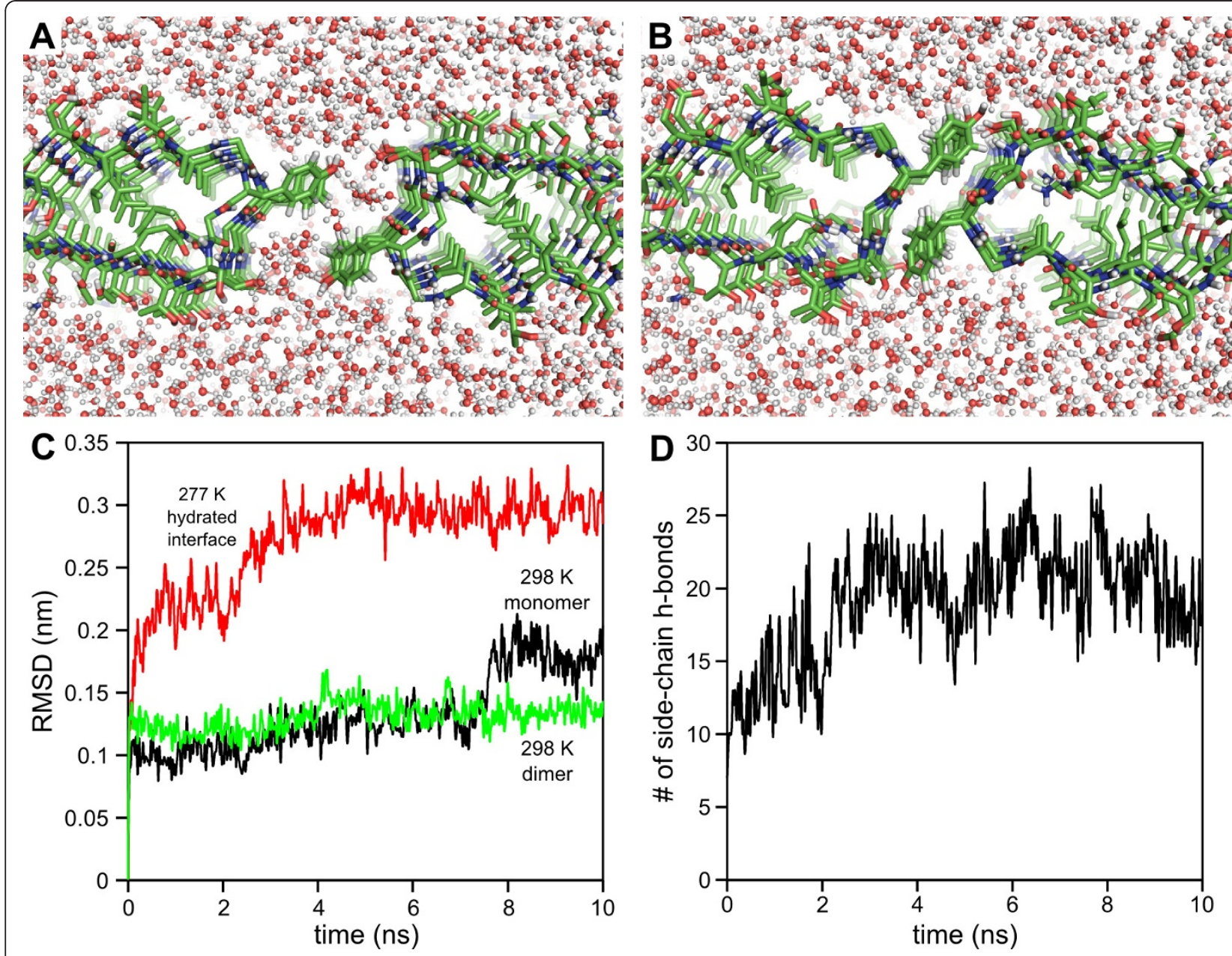

Figure 5 MD simulations of the PbINP dimer. A) Two PbINP monomers were aligned via their tyrosine ladders prior to the start of the MD simulation. Waters allowed to equilibrate at the dimerization interface prior to the start of the simulation are shown as red and white spheres. B) Final scene from the 10-ns simulation showing exclusion of waters from the dimerization interface. C) The C $\alpha$ RMSD (nm) of the PbINP dimer was plotted as a function of time (ns). The plots are of either the dimer starting from the hydrated interface (red line) or the energy-minimized average dimer simulated at the elevated temperature of $298 \mathrm{~K}$ (green line). A plot of the C $\alpha$ RMSD $(\mathrm{nm})$ of the PbINP monomer (black line) run at the elevated temperature of $298 \mathrm{~K}$ was also plotted as a function of time (ns). D) The number of side-chain hydrogen bonds formed during the simulation was plotted as a function of time (ns). 


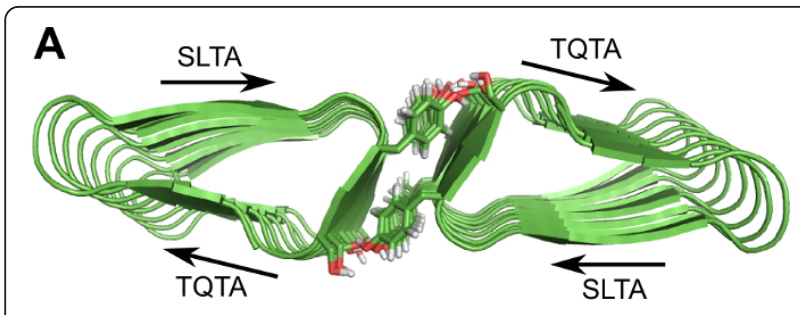

B

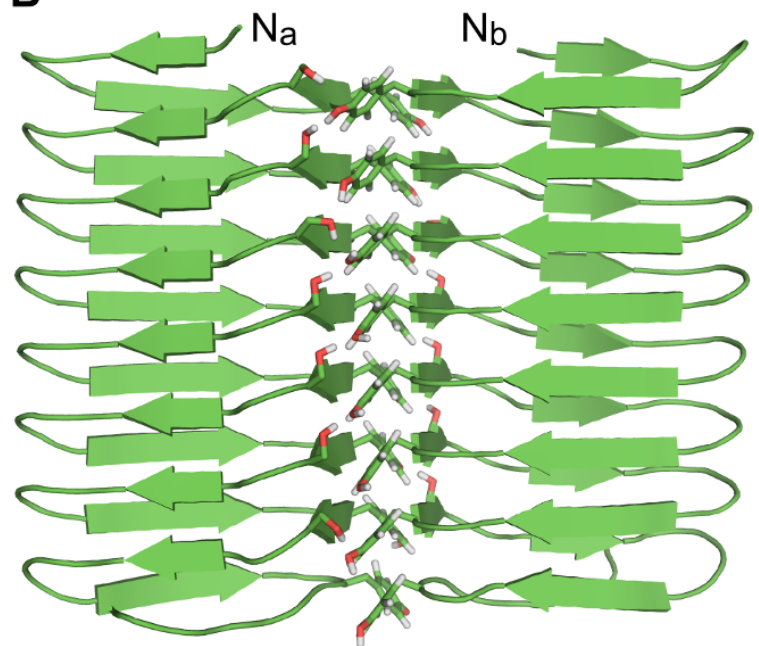

Figure 6 Dimerization interface of PbINP. A) Cross-section endon view of the energy-minimized average PbINP dimeric structure. The protein is shown in cartoon format, with the tyrosine and serine residues that constitute the interface shown in stick format. The SLTA and TQTA active sites of each chain are indicated. The colouring scheme is the same as in Figure 2. B) Top-down view of the PbINP dimer. The $\mathrm{N}$ termini of the $\mathrm{a}$ and $\mathrm{b}$ chains that constitute the dimer are indicated.

dimeric structure at the elevated temperatures of $298 \mathrm{~K}$ and $310 \mathrm{~K}$ (Figure 5C, Additional file 1 - Figure S4). In each case, the dimer remained stable throughout the trajectory, with no dissociation of individual chains, and complete exclusion of water molecules at the dimerization interface. Identical MD simulations were performed on the PbINP monomer, and in each case, a partial unravelling of the $\mathrm{C}$ terminus of the protein occurred (at the 8-ns mark of the $298 \mathrm{~K}$ simulation and the 4-ns mark of the $310 \mathrm{~K}$ simulation) (Figure 5C, see Additional file 1 - Figure S4). These results demonstrate that dimerization increases the stability of PbINP.

We also attempted to model PbINP as an anti-parallel dimer (see Additional file 1, Figure S5). The model remained intact following a 10-ns solvated MD simulation, however, a twist developed in the structure that did not develop in the parallel dimer (Figures 6, 7). In an anti-parallel orientation, the hydroxyl groups of the solvent-exposed rank of serine residues from one chain are unable to form an intercalated hydrogen-bond network with the hydroxyl groups of the tyrosine residues of the opposite chain. This lack of interaction allowed the twist to occur and therefore spoiled the flatness of the dimer. Flatness is a defining characteristic of icebinding proteins, and as such, we think it is unlikely that PbINP would dimerize in an anti-parallel manner.

The ice nucleating surfaces of PbINP are highly conserved $A$ s previously mentioned, when PbINP is modelled as a parallel dimer, the SLTA surface of one chain resides on the same side of the dimer as the TQTA surface of the other chain (Figure 6A). This creates a flat surface on both sides of the dimer, each spanning the entire width of the structure. The amino acid composition of both the TQTA and SLTA surfaces is highly conserved, as is the area in between them created by the dimerization interface. More specifically, the solvent-exposed first and third position of the TQTA motifs have threonines present at these positions $87 \%$ and $92 \%$ of the time respectively. The solventexposed first and third position of the SLTA motifs show greater variation, however, serines and threonines are present at the first position $71 \%$ of the time (48\% serine, $23 \%$ threonine), while threonine and isoleucine are present at the third position $98 \%$ of the time (60\% threonine, $38 \%$ isoleucine). The rare substitutions at these positions are aspartate, valine and leucine. The dimerization interface is the most highly conserved portion of the whole protein, with the intercalated hydroxyl groups of the tyrosine and serine residues present $90 \%$ and $98 \%$ of the time, respectively.

\section{PbINP orders water molecules via the anchored clathrate water mechanism}

This high degree of amino acid conservation endows the PbINP dimer with two sides that are flat, and relatively hydrophobic, but also contain many hydrogen bond donors and acceptors. These characteristics define the IBS's of all AFPs, and as such, raise the possibility that INPs function by the ACW mechanism of AFP action [13]. The ACW mechanism states that the relative hydrophobicity of an AFP's IBS orders water molecules into an ice-like lattice, and this lattice is then anchored to the surface of the protein via hydrogen bonds.

To investigate the water ordering potential of PbINP, a 2-ns MD simulation was performed on the energyminimized average dimeric structure at a temperature of $273 \mathrm{~K}$. The TIP5P water model was used as it accurately represents the behaviour of water during an MD simulation $[27,28]$. Waters within $10 \AA$ of $P b I N P$ 's surface were extracted at 20-ps intervals throughout the course of the simulation and their crystallographic structure factors were calculated followed by a Fourier transform, giving the electron density of all extracted waters. Strong electron density with ice-like spacing was present across the entire width of the protein's surface (Figure 7A). Waters built into this density aligned down the 


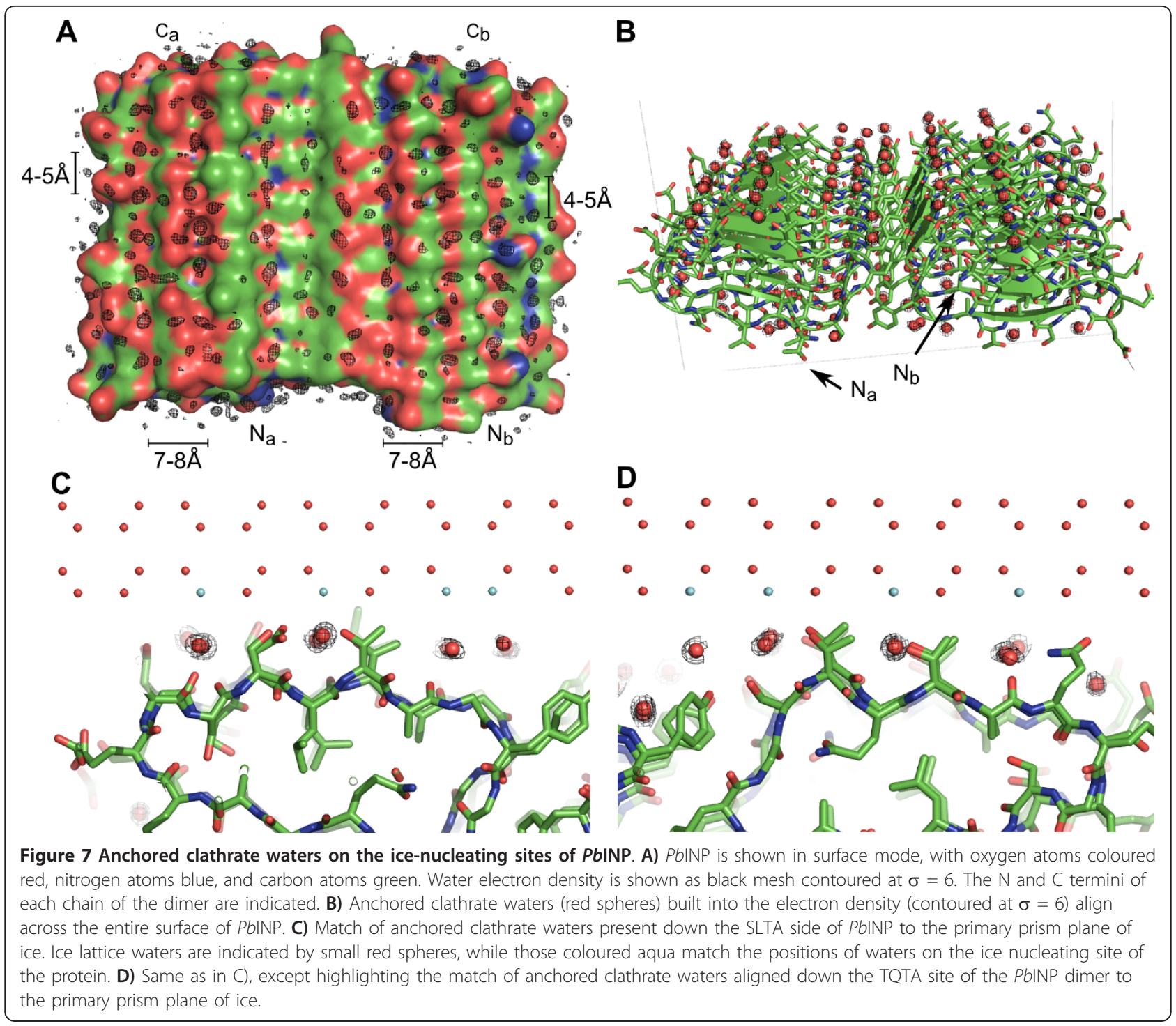

troughs created by the outward projecting residues of the TQTA and SLTA motifs, down the flat areas immediately preceding and following these motifs, and across the dimerization interface as well (Figure $7 \mathrm{~B}$ ). These waters closely match the $4.5 \AA \times 7.35 \AA$ spacing of waters on the primary prism plane of ice, and as such, have revealed a potential orientation of a nascent ice crystal on the surface of the INP (Figures 7C,D).

As a positive control, the same simulation was performed on MpAFP_RIV, a hyperactive $\mathrm{Ca}^{2+}$-dependent $\beta$-helical AFP produced by the Antarctic bacterium Marinomonas primoryensis. MpAFP_RIV was chosen as the positive control because the positions of the ACWs on its IBS are known [13]. The simulation accurately predicted the position of the ice-like waters on the IBS of the protein (see Additional file 1, Figure S6A,B). Indeed, aligning 22 manually-built waters to their crystallographic counterparts on the IBS of chain B of MpAFP_RIV produced an RMSD of only $0.51 \AA$. Further proof of the accuracy of the MD simulation is evidenced by the identical coordination of waters present down the trough created by the TQTA motifs of $P b I N P$ as compared to the waters located down the IBS TxT troughs of the $\beta$-helical insect AFPs (TmAFP and sbwAFP) as revealed by $\mathrm{X}$-ray crystallography (Figure 8 ).

\section{Dimerization increases the active surface area of PbINP}

The temperature at which an INP nucleates ice is dependent upon its oligomerization state [4]. Approximately one INP monomer is capable of nucleating ice at a temperature of $-12^{\circ} \mathrm{C}$, while an aggregate of at least 50 INP monomers is required for ice nucleation in the $-2^{\circ} \mathrm{C}$ to $-3^{\circ} \mathrm{C}$ temperature range. However, due to the lack of an experimentally-determined INP structure, the mechanism by 


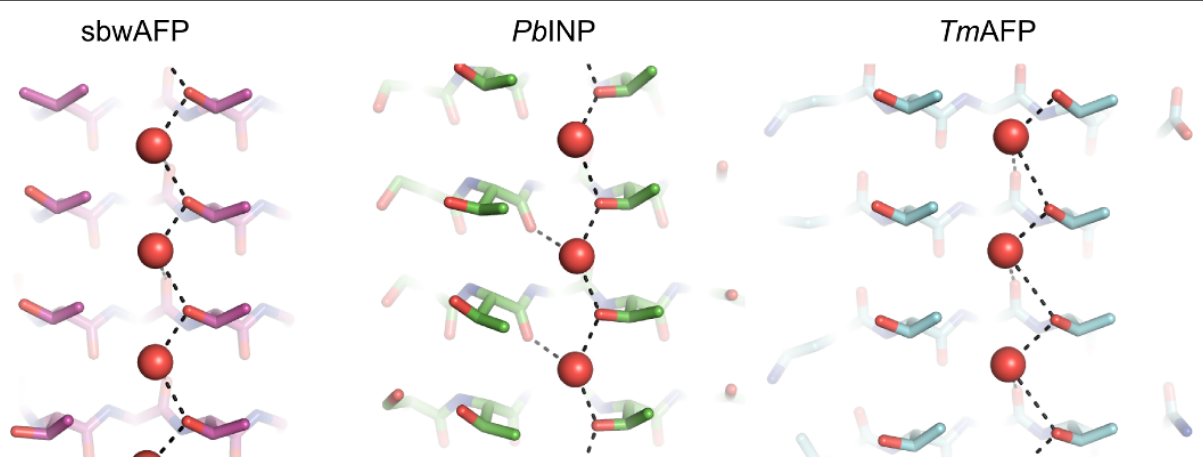

Figure 8 Anchored clathrate water comparison. Waters located down the TQTA trough of PbINP (middle image) show near identical coordination as compared to the ice-like waters located down the TXT troughs of TmAFP (PDB 1EZG (chain A)) (right image) and sbwAFP (PDB $1 \mathrm{M} 8 \mathrm{~N}$ (chain A)) (left image) as revealed by X-ray crystallography. Hydrogen bonds are represented by black hatched lines, while oxygens are coloured red, and nitrogens dark blue. Waters are represented as red spheres.

which an INP oligomerizes has remained a mystery. Previously, there have been only two attempts to predict how an INP might oligomerize. Wu et al. [12] speculated that overlapping protein-protein interactions between the two flat IBS motifs could generate 'stairs' of INPs, facilitating ice growth along the discontinuities. Prior to that, however, Kajava and Lindow [10] modelled PsINP as an array of interdigitating anti-parallel $\beta$-strands that formed a flat ice-nucleating array upon oligomerization. While intriguing, the model was created prior to the structural determination of several $\beta$-helical AFPs [13-15], and as such, it overlooked the potential water-organizing capabilities of the TQTA and SLTA motifs when aligned as parallel $\beta$ strands in a flat $\beta$-sheet. As previously mentioned, Graether and Jia [6] modelled an INP as a $\beta$-helix, but the potential for oligomerization was not discussed. In that model, the serine of the GYGS motif pointed inwards and was not solvent exposed as predicted in this study. This would prevent formation of the intercalated hydrogenbond network between the serine and tyrosine residues of the GYGS motifs from opposing chains in the dimer. Nevertheless, both $\beta$-helical models predict a solventexposed orientation for the tyrosine residue of the GYGS motif, and this argues against INP peptide studies that suggest it points towards the interior of the structure $[8,9]$. It is likely that short peptides adopt conformations in solution that are not representative of the full-length protein.

As a dimer with two bridged active sites on opposite sides of the structure, the potential ice-nucleating surface area of PbINP is dramatically increased (Figure 9). A full-length $P b I N P$ dimer would have an active surface area of ca. $25,600 \AA^{2}(40 \AA \times 320 \AA \times 2)$, a value slightly larger than the $20,100 \AA^{2}$ minimum surface area required for an ice embryo to continue growing at $-12^{\circ} \mathrm{C}[29]$ (the temperature at which a single INP molecule is active [4]). By dimerizing in an offset manner, PbINP monomers could form extended oligomeric structures that would increase the active surface area of the complex without occluding it, therefore raising the temperature at which ice nucleation occurs (Figure 10). In this scenario, the repetitive central domain of each $P b I N P$ chain runs parallel to the surface of the bacterium, with the non-repetitive $\mathrm{N}$ terminus of each chain serving as a membrane anchor. Multiple arrays of offset dimers could increase the active PbINP surface area on the outer membrane of the bacterium. Following initial construction of the monomeric model of PbINP, it was thought the solvent-exposed tyrosine ladder might play a role in anchoring the protein to the membrane. However, several studies have shown the $\mathrm{N}$-terminal domain alone is capable of performing this task [30,31], and it therefore would seem redundant to have other segments of the protein anchored to the bacterium, which would restrict the protein's access to solvent. Further investigation into the tertiary and quaternary structures of bacterial INPs, both in vitro and in vivo, are required and this oligomerization scenario is just one theoretical way of extending the ice nucleation surface.

Even without oligomerization, the size of PbINP is significantly larger than any known AFP (Figure 9). This fact, combined with an INP's ability to order water molecules via the ACW mechanism, further strengthens the idea that size, and not a particular protein fold or mechanism of action, is the primary discriminating factor between the two families of proteins with diametrically opposite functions.

\section{Conclusions}

This paper demonstrates through the use of homology modelling and MD simulations that bacterial INPs are able to fold as novel $\beta$-helical dimers. De-solvation of solvent-exposed tyrosine ladders drives INP dimerization, and this allows the ice nucleation sites of the protein to extend as a continuum across the width of the 


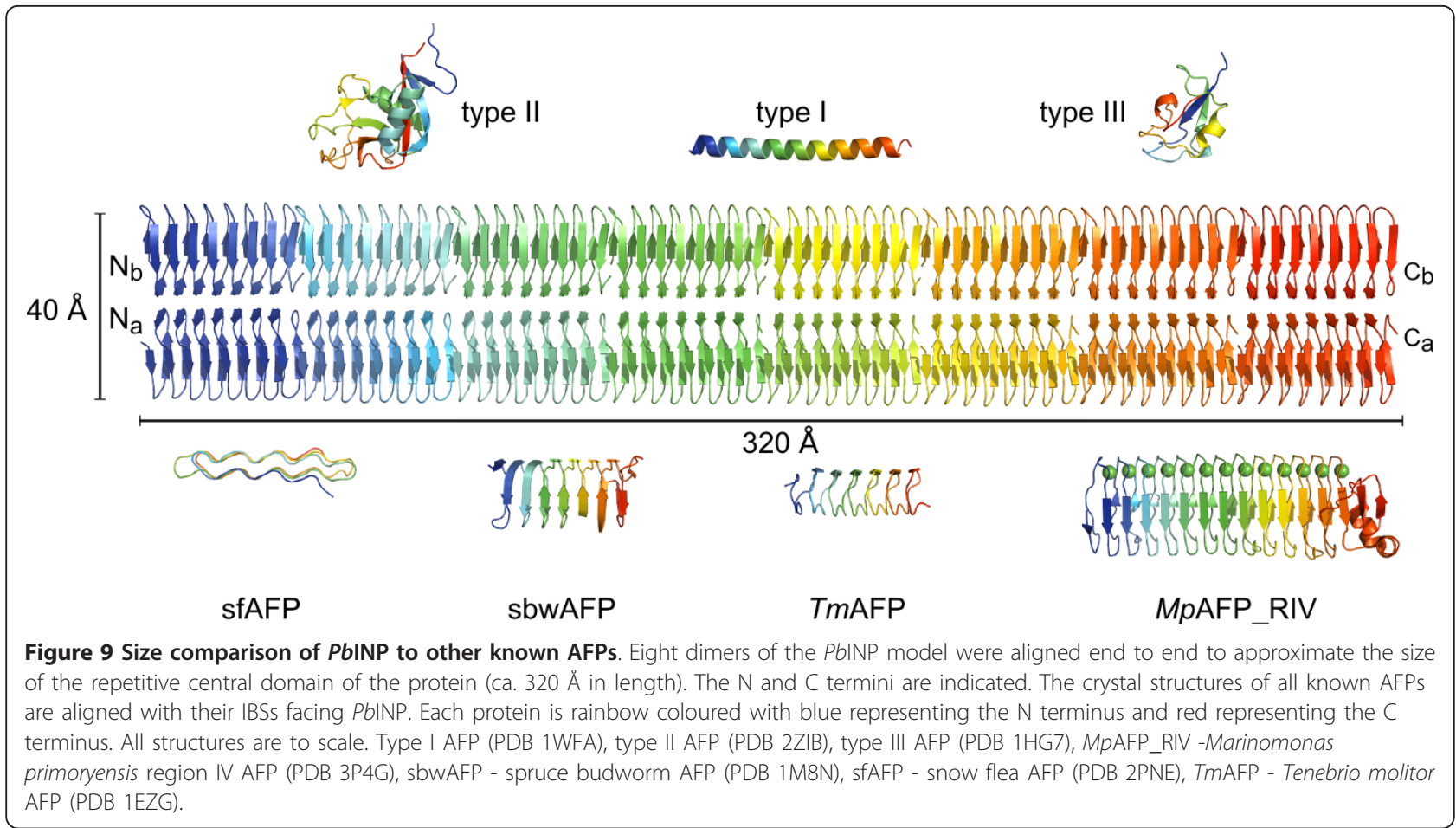

dimer. Both sides of the dimer order water molecules into an ice-like lattice using the anchored clathrate water mechanism of action. Offset dimerization can allow INP oligomerization without active site occlusion. This increases the active surface area of the INP, therefore raising the temperature at which ice nucleates.

\section{Methods}

\section{Initial model building}

All model building was performed using a combination of the programs PyMOL [32] and SYBYL (version 6.4, Tripos Associates, St. Louis, MO). PyMOL was used to excise the $\beta$-roll from alkaline protease (PDB 1KAP), remove the roll's $\mathrm{Ca}^{2+}$-binding turns, and change its tripeptide $x u x \beta$-strands (where $\mathrm{x}$ is any amino acid and $\mathrm{u}$ is a hydrophobic residue) to the appropriate TQTA and SLTA motifs of PbINP. SYBYL was used to manually build and energetically minimize the $\mathrm{xxxS}$ and GYGS loops that connect the TQTA and SLTA $\beta$-strands. Finally, PyMOL was used to copy and expand the 16-aa loop until an 8-loop $\beta$-helix was completed (corresponding to residues 217-345 of $\mathrm{PbINP}$ ).

\section{Molecular Dynamics simulations of initial model}

All MD simulations were performed using the program Gromacs v. 4.5.3 [33]. Prior to all full-scale MD

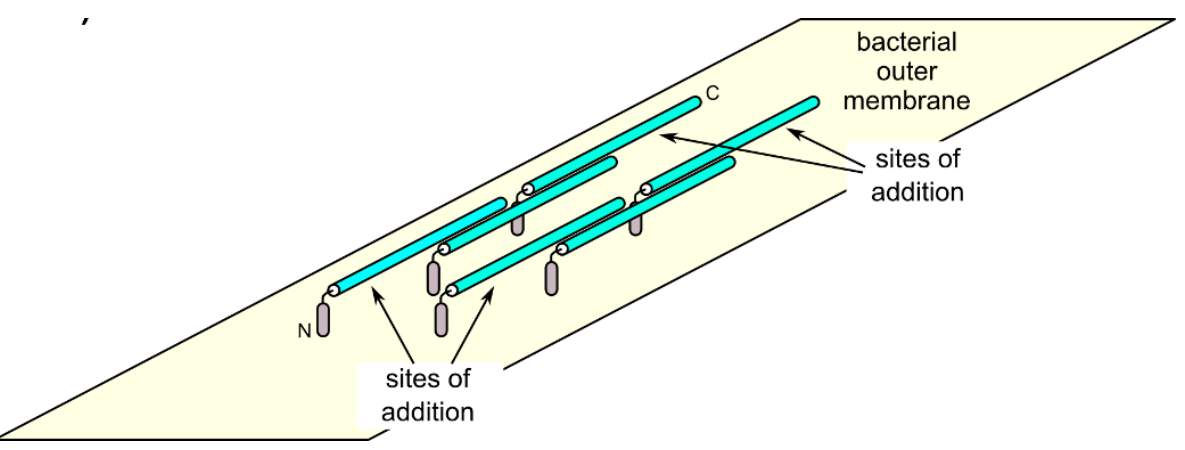

Figure 10 Offset PbINP dimerization about the surface of $P$. borealis. PbINP monomers are shown as individual tubes, with the highly repetitive central domains coloured light blue and the non-repetitive $N$ termini coloured grey. The $N$ terminus of the first PbINP chain of one multimer is indicated, as is the $C$ terminus of the last PbINP chain of the same multimer. To increase active surface area, individual PbINP chains could add to the exposed sections near the two termini. 
simulations described below, energy minimization and a 50-ps position-restrained MD simulation was performed to relax the solvent around the protein. Berendsen temperature and pressure coupling were applied in all cases, and the GROMOS96 43a1 force field and SPC water model were used unless stated otherwise. The initial model of PbINP (residues 217-345) was solvated in a box containing 6239 water molecules and $14 \mathrm{Na}^{+}$ions to offset the charge of the protein. A full-scale 5-ns MD simulation was then performed at $277 \mathrm{~K}$. The average structure of the final $3 \mathrm{~ns}$ of the simulation was calculated and energy minimized in vacuo. A Ramachandran plot of the protein was generated using the program PROCHECK [34].

\section{PbINP dimer construction and MD simulations}

The dimer of PbINP was built by duplicating the protein, rotating it $180^{\circ}$ about the long axis of the structure, and then placing the duplicated chain's tyrosine ladder in close proximity to the tyrosine ladder of the original chain. This parallel arrangement of the helices placed their $\mathrm{N}$ termini at the same end of the dimer. The dimer was solvated in a box containing 13,401 waters and $28 \mathrm{Na}^{+}$ions to offset the charge of the protein. The protein was subjected to a 10 -ns full-scale MD simulation. An average structure of the protein from the final $5 \mathrm{~ns}$ of the simulation was calculated and energy minimized in the same manner as previously mentioned. This structure was then re-solvated and subjected to a 10 -ns full-scale MD simulation at $298 \mathrm{~K}$ to test the stability of the dimeric protein. A single chain from the dimer was also subjected to the same simulation to test its stability at the elevated temperature of $298 \mathrm{~K}$.

\section{PbINP dimer hydration studies}

A 2-ns MD simulation was performed at the temperature of $273 \mathrm{~K}$ on the energy-minimized average dimeric structure of $P b I N P$ to investigate the hydration of the protein. As a positive control, chain B from region IV of the AFP produced by the Antarctic bacterium Marinomonas primoryensis (MpAFP_RIV) (PDB 3P4G) was subjected to the same simulation. The OPLS-aa force field $[35,36]$ along with the TIP5P water model [27] were used in each case. To determine the probability density of water molecules, the following protocol was followed for each MD simulation. Coordinates of the system were extracted at 20-ps intervals throughout the course of the simulation (100 coordinate sets total/simulation). After superimposing these 100 structures by performing a least-squares fit on the protein $\mathrm{C} \alpha$ atoms, the waters within $10 \AA$ of the protein were extracted and their electron densities were determined by calculating crystallographic structure factors followed by a Fourier transform using the programs SFall [37] and FFT [38] respectively of the CCP4 software suite [39]. The electron densities were calculated with the protein and water in a P1 unit cell equivalent to the box size used for the MD simulation. The FFT calculation used a grid spacing of $0.33 \AA$ and a resolution of $1 \AA$. Waters were then manually built into the density using the program Coot [40].

\section{Additional material}

Additional file 1: Supplementary Information. Supplementary Figures $1,2,3,4,5,6$.

\section{Abbreviations}

aa: amino acid; ACW: anchored clathrate water; AFP: antifreeze protein; IBS: ice-binding site; INP: ice nucleation protein; MD: molecular dynamics; MpAFP_RIV: Marinomonas primoryensis region IV antifreeze protein; PbINP: Pseudomonas borealis ice nucleation protein; PsINP: Pseudomonas syringae ice nucleation protein; sbwAFP: spruce budworm antifreeze protein; sfAFP snow flea antifreeze protein; TmAFP: Tenebrio molitor antifreeze protein.

\section{Acknowledgements}

We are grateful to Dr. John Allingham for access to his servers. This work was funded by grants from the CIHR and NSERC to PLD and VKW, respectively. CPG was the recipient of an NSERC-PGSD3 scholarship and an R. Samuel McLaughlin fellowship. PLD holds a Canada Research Chair in Protein Engineering and VKW is a Queen's University Research Chair.

\section{Author details}

'Department of Biochemistry, Queen's University, Kingston, ON, K7L-3N6, Canada. ${ }^{2}$ Department of Biology, Queen's University, Kingston, ON, K7L-3N6, Canada. ${ }^{3}$ Department of Microbiology and Immunology, Queen's University, Kingston, ON, 7L-3N6, Canada.

\section{Authors' contributions}

CPG performed all modelling and molecular dynamics simulations. The manuscript was written by CPG with editorial input from RLC, VKW, and PLD. All authors read approved the final manuscript.

Received: 23 June 2011 Accepted: 27 September 2011 Published: 27 September 2011

\section{References}

1. Jia Z, Davies PL: Antifreeze proteins: an unusual receptor-ligand interaction. Trends Biochem Sci 2002, 27:101-106.

2. Lindow SE, Arny DC, Upper CD: Bacterial ice nucleation: a factor in frost. injury to plants. Plant Physiol 1982, 70:1084-1089.

3. Warren $G$, Wolber P: Molecular aspects of microbial ice nucleation. Mol Microbiol 1991, 5:239-243.

4. Govindarajan AG, Lindow SE: Size of bacterial ice-nucleation sites measured in situ by radiation inactivation analysis. Proc Natl Acad Sci USA 1988, 85:1334-1338.

5. Warren $\mathrm{G}$, Corotto $\mathrm{L}$ : The consensus sequence of ice nucleation proteins from Erwinia herbicola, Pseudomonas fluorescens and Pseudomonas syringae. Gene 1989, 85:239-242.

6. Graether SP, Jia Z: Modeling Pseudomonas syringae ice-nucleation protein as a beta-helical protein. Biophys J 2001, 80:1169-1173.

7. Ala P, Chong P, Ananthanarayanan VS, Chan N, Yang DS: Synthesis and characterization of a fragment of an ice nucleation protein. Biochem Cell Biol 1993, 71:236-240.

8. Kumaki Y, Kawano K, Hikichi K, Matsumoto T, Matsushima N: A circular loop of the 16-residue repeating unit in ice nucleation protein. Biochem Biophys Res Commun 2008, 371:5-9.

9. Tsuda S, Ito A, Matsushima N: A hairpin-loop conformation in tandem repeat sequence of the ice nucleation protein revealed by NMR spectroscopy. FEBS Lett 1997, 409:227-231. 
10. Kajava AV, Lindow SE: A model of the three-dimensional structure of ice nucleation proteins. J Mol Biol 1993, 232:709-717.

11. Kobashigawa Y, Nishimiya Y, Miura K, Ohgiya S, Miura A, Tsuda S: A part of ice nucleation protein exhibits the ice-binding ability. FEBS Lett 2005, 579:1493-1497.

12. Wu Z, Qin L, Walker VK: Characterization and recombinant expression of a divergent ice nucleation protein from 'Pseudomonas borealis'. Microbiology 2009, 155:1164-1169.

13. Garnham CP, Campbell RL, Davies PL: Anchored clathrate waters bind antifreeze proteins to ice. Proc Natl Acad Sci USA 2011, 108:7363-7367.

14. Graether SP, Kuiper MJ, Gagné SM, Walker VK, Jia Z, Sykes BD, Davies PL: Beta-helix structure and ice-binding properties of a hyperactive antifreeze protein from an insect. Nature 2000, 406:325-328.

15. Liou YC, Tocilj A, Davies PL, Jia Z: Mimicry of ice structure by surface hydroxyls and water of a beta-helix antifreeze protein. Nature 2000, 406:322-324.

16. Kuiper MJ, Davies PL, Walker VK: A theoretical model of a plant antifreeze protein from Lolium perenne. Biophys J 2001, 81:3560-3565.

17. Middleton AJ, Brown AM, Davies PL, Walker VK: Identification of the icebinding face of a plant antifreeze protein. FEBS Lett 2009, 583:815-819.

18. Garnham CP, Gilbert JA, Hartman CP, Campbell RL, Laybourn-Parry J, Davies PL: A Ca2+-dependent bacterial antifreeze protein domain has a novel beta-helical ice-binding fold. Biochem J 2008, 411:171-180.

19. Wen $D$, Laursen RA: A D-antifreeze polypeptide displays the same activity as its natural L-enantiomer. FEBS Lett 1993, 317:31-34.

20. Pentelute BL, Gates ZP, Tereshko V, Dashnau JL, Vanderkooi JM, Kossiakoff $A A$, Kent SBH: X-ray structure of snow flea antifreeze protein determined by racemic crystallization of synthetic protein enantiomers. J Am Chem Soc 2008, 130:9695-9701.

21. Hennetin J, Jullian B, Steven AC, Kajava AV: Standard conformations of beta-arches in beta-solenoid proteins. J Mol Biol 2006, 358:1094-1105.

22. Kajava AV, Steven AC: Beta-rolls, beta-helices, and other beta-solenoid proteins. Adv Protein Chem 2006, 73:55-96.

23. Kajava AV, Steven AC: The turn of the screw: variations of the abundant beta-solenoid motif in passenger domains of Type $\mathrm{V}$ secretory proteins. J Struct Biol 2006, 155:306-315.

24. Bublitz M, Holland C, Sabet C, Reichelt J, Cossart P, Heinz DW, Bierne H, Schubert W: Crystal structure and standardized geometric analysis of InIJ, a listerial virulence factor and leucine-rich repeat protein with a novel cysteine ladder. J Mol Biol 2008, 378:87-96.

25. Biancalana M, Makabe K, Koide A, Koide S: Aromatic cross-strand ladders control the structure and stability of beta-rich peptide self-assembly mimics. J Mol Biol 2008, 383:205-213.

26. Biancalana M, Makabe K, Koide S: Minimalist design of water-soluble cross-beta architecture. Proc Natl Acad Sci USA 2010, 107:3469-3474.

27. Mahoney MW, Jorgensen $\mathrm{WL}$ : A five-site model for liquid water and the reproduction of the density anomaly by rigid, nonpolarizable potential functions. Journal of Chemical Physics 2000, 112:8910-8922

28. Nutt DR, Smith JC: Dual function of the hydration layer around an antifreeze protein revealed by atomistic molecular dynamics simulations. J Am Chem Soc 2008, 130:13066-13073.

29. Hobbs PV: Ice Physics. Clarendon Press, Oxford; 1974

30. van Bloois E, Winter RT, Kolmar H, Fraaije MW: Decorating microbes: surface display of proteins on Escherichia coli. Trends Biotechnol 2011, 29:79-86.

31. Li Q, Yu Z, Shao X, He J, Li L: Improved phosphate biosorption by bacterial surface display of phosphate-binding protein utilizing ice nucleation protein. FEMS Microbiol Lett 2009, 299:44-52.

32. DeLano WL: The PyMOL Molecular Graphics System. DeLano Scientific, San Carlos, CA; 2002 [http://www.pymol.org]

33. Van Der Spoel D, Lindahl E, Hess B, Groenhof G, Mark AE, Berendsen HJC: GROMACS: fast, flexible, and free. J Comput Chem 2005, 26:1701-1718.

34. Laskowski RA, Rullmannn JA, MacArthur MW, Kaptein R, Thornton JM: AQUA and PROCHECK-NMR: programs for checking the quality of protein structures solved by NMR. J Biomol NMR 1996, 8:477-486.

35. Jorgensen WL, Maxwell DS, Tirado-Rives J: Development and Testing of the OPLS All-Atom Force Field on Conformational Energetics and Properties of Organic Liquids. Journal of the American Chemical Society 1996, 118:11225-11236.

36. Kaminski GA, Friesner RA, Tirado-Rives J, Jorgensen WL: Evaluation and Reparametrization of the OPLS-AA Force Field for Proteins via
Comparison with Accurate Quantum Chemical Calculations on Peptides. The Journal of Physical Chemistry B 2001, 105:6474-6487.

37. Agarwal RC: A new least-squares refinement technique based on the fast Fourier transform algorithm. Acta Crystallographica Section A 1978, A34:791-809.

38. Read RJ, Schierbeek AJ: A phased translation function. Journal of Applied Crystallography 1988, 21:490-495.

39. Winn MD, Ballard CC, Cowtan KD, Dodson EJ, Emsley P, Evans PR, Keegan RM, Krissinel EB, Leslie AGW, McCoy A, et al: Overview of the CCP4 suite and current developments. Acta Crystallogr D Biol Crystallogr 2011, 67:235-242.

40. Emsley P, Cowtan K: Coot: model-building tools for molecular graphics. Acta Crystallogr D Biol Crystallogr 2004, 60:2126-2132.

41. Crooks GE, Hon G, Chandonia J, Brenner SE: WebLogo: a sequence logogenerator. Genome Res 2004, 14:1188-1190.

doi:10.1186/1472-6807-11-36

Cite this article as: Garnham et al:: Novel dimeric $\beta$-helical model of an ice nucleation protein with bridged active sites. BMC Structural Biology 2011 11:36.

\section{Submit your next manuscript to BioMed Central and take full advantage of:}

- Convenient online submission

- Thorough peer review

- No space constraints or color figure charges

- Immediate publication on acceptance

- Inclusion in PubMed, CAS, Scopus and Google Scholar

- Research which is freely available for redistribution

Submit your manuscript at www.biomedcentral.com/submit
C) Biomed Central 